\title{
Las podas de roble (Quercus pyrenaica) en la Comarca de Almarza (Soria). Documentación de la experiencia, situación actual y perspectivas de futuro
}

\section{The oak (Quercus pyrenaica) pruning in the region of Almarza (Soria). Documentation of the experience, current situation and future perspectives}

\author{
Alcalde Olivares, C." \\ Servicio Territorial de Medio Ambiente de Soria. Junta de Castilla y León. c/ Los Linajes, 1; 42071 - Soria \\ *Autor para correspondencia: alcolima@jcyl.es
}

\section{Resumen}

En el norte de la provincia de Soria, con montes públicos de aprovechamiento comunal, se ha mantenido desde antiguo el aprovechamiento de leña en las masas adehesadas sobre Quercus pyrenaica fundamentalmente (y otros Quercus, como Q. petraea y $Q$. faginea). Las podas se realizan mediante el trasmocho, y permiten compatibilizar la producción de leña y pasto en la misma superficie. A pesar de haberse reducido drásticamente los volúmenes de leña vecinal demandados en los pueblos, se ha conservado y transmitido la forma de ejecutar estas podas, e incluso en los últimos años se observa un aumento significativo de la demanda de leña. En otras zonas cercanas esta forma de aprovechamiento ha dejado de realizarse, y encontramos ahora árboles, en algunos casos monumentales, con graves signos de decaimiento y riesgo de desgarro, por no haber sido podados a tiempo. Con objeto de documentar las prácticas tradicionales, y basándose en la experiencia de técnicos y agentes medioambientales, se ha elaborado un manual de poda muy sencillo, para poder exportar el modelo a otras zonas en las que ya se ha perdido esa forma de aprovechamiento. Se trata de un documento asequible a todo tipo de personas vinculadas a las cortas de leña. Además se están realizando experiencias con variaciones sobre el trasmocho "tipo" para estudiar las diferentes respuestas de los árboles.

\section{Summary}

In the north of the province of Soria, with public forests of communal use, it has been maintained since ancient times the use of firewood in the masses mainly on Quercus pyrenaica (and other Quercus, such as $Q$. petraea and $Q$. faginea). The pruning is done by pollarding, and allows the production of firewood and grass on the same surface. Despite having drastically reduced the volumes of local firewood demanded in the villages, the way to execute these prunings has been conserved and transmitted, and even in recent years there has been a significant increase in the demand for firewood. In other nearby areas this form of use has ceased, and we now find trees, in some cases monumental, with serious signs of decay and risk of tearing, for not having been pruned in time. In order to document traditional practices, and based on the experience of technicians and environmental agents, a very simple pruning manual has been developed, in order to export the model to other areas where this form of use has already been lost. It is an affordable document for all types of people linked to firewood cutting. In addition, experiments are being carried out with variations on the "type" pollard to study the different responses of the trees.

Palabras clave: Comarca del Valle, dehesa, leña, trasmocho.

Keywords: Comarca del Valle, pasture, firewood, pollarding. 


\section{Introducción}

La Comarca del Valle, que se corresponde con la comarca forestal de Almarza, se encuentra en el norte de la provincia de Soria, enmarcada por la Sierra Cebollera y la Sierra de Montes Claros. Se corresponde con las cuencas del Río Tera y su afluente, el Río Razón, y el tramo del Río Duero entre Vinuesa y Soria.

En esta comarca, cada localidad cuenta con su monte de utilidad pública. La propiedad de los terrenos corresponde a las Entidades Locales, y ellas adjudican tradicionalmente los aprovechamientos a los vecinos, basándose en ordenanzas y costumbres.

Como ejemplo de ello, existe un arca muy antigua en la que se conserva la documentación relativa al monte de utilidad pública n. ${ }^{\circ} 106$ del Catálogo de UP de la provincia de Soria, denominado "Dehesa Mata", propiedad del Ayuntamiento de Almarza y del antiguo Ayuntamiento de San Andrés de Soria, hoy integrado en el anterior. En ella se recogen todos los documentos que hacen referencia a la gestión comunal de la Dehesa y de los diferentes pleitos que el pueblo de San Andrés de Soria y Almarza han mantenido durante siglos con diferentes instituciones, para defender los derechos de sus vecinos. Según la documentación de esta arca, en 1329 ya se envía una carta a Alfonso XI para que se respete el derecho de uso privativo de la Dehesa llamada La Mata. La primera ordenanza del monte fue aprobada por Carlos I, y los vecinos de ambos pueblos (y otros dos, ya desaparecidos) tenían derecho a pastos a cambio de colaborar en las labores de mantenimiento (Martínez Rodríguez, 2014).

En la actualidad, los aprovechamientos principales de estos montes son los de pastos y leñas. El aprovechamiento vecinal de pastos es residual, cuando no inexistente, y los pastos sobrantes se adjudican directamente a los vecinos ganaderos del pueblo. La presión ganadera es elevada, pues son zonas en las que éste es uno de los pocos recursos económicos que existen.

El aprovechamiento de leñas, sin embargo, sigue siendo vecinal en su mayoría, y está muy arraigado el derecho de la comunidad de obtener la leña para el consumo de sus hogares. Durante varias décadas, la demanda de leñas disminuyó drásticamente debido al despoblamiento rural y a la calefacción de gasoil, pero en los últimos 810 años se está experimentando un repunte por diversos factores, entre los que se encuentran la crisis económica, la subida del precio del petróleo, la mejora de la eficiencia de las calderas y una mayor conciencia ecológica.

La forma óptima de compatibilizar los aprovechamientos de pastos y de leñas en una misma superficie es mediante el trasmocho de los árboles. La poda permite elevar el monte bajo a unos 3 metros de altura, con lo que queda fuera del diente del ganado. Por eso, actualmente estos montes son dehesas principalmente de roble (como se llama a Quercus pyrenaica en esta zona de Castilla), acompañado de quejigo ( $Q$. faginea), roble albar $(Q$. petraea) y fresno (Fraxinus angustifolia), con árboles trasmochos, algunos de gran envergadura. El trasmocho es un tipo de poda cuyo objetivo es la producción periódica de leñas, a diferencia de otras podas. Estas formaciones gozan de una altísima biodiversidad, y muchos de estos montes se encuentran además en los ZEC "Sierras de Urbión y Cebollera" y "Robledales del Berrún". 
El inconveniente principal de esta forma de masa es que es muy artificial, y que precisa de una periodicidad en los tratamientos. El abandono de los trabajos de trasmocho en estos árboles suele conllevar un envejecimiento acelerado de su fisiología (Cantero y Passola, 2013). Las ramas de los robles viejos que se han dejado de podar alcanzan unos pesos descomunales, que en algunos casos llegan a descuajarse y provocan graves desgarros. Por eso es preciso continuar podando, para evitar la muerte de estos árboles monumentales.

Las dehesas de trasmochos son un paisaje cultural. Son el fruto del manejo del ecosistema por parte de varias generaciones para la obtención de leña, compatibilizándolo con la existencia del propio árbol y con el aprovechamiento ganadero. La necesidad de mantener la cultura del trasmocho resulta clave para la conservación de los paisajes tradicionales (Allende et al., 2013). Actualmente, existe un movimiento en pro de la protección, gestión y conservación de los paisajes, motivado por el convencimiento de que éste es un elemento importante para la calidad de vida individual y social y para la actividad económica. El hecho de que la dehesa de trasmochos sea un paisaje cultural hace necesario que los árboles sean nuevamente manejados. De no hacerlo, les llegará antes de tiempo su senescencia, muerte y desaparición (Francisco, 2013).

Existen otras formas de poda características de otras zonas de España, como por ejemplo las podas a horca y pendón, que se empleaban para obtener grandes escuadrías con destino naval (Aragón Ruano, 2009). Sin embargo, el modo de podar en los pueblos de la Comarca del Valle se caracteriza por favorecer al máximo la producción de leña de pequeñas dimensiones.

Las podas de roble han seguido realizándose en la mayoría de los pueblos a cargo de los propios vecinos. Como es un aprovechamiento con cierta dificultad de ejecución, en ocasiones son solo unos pocos los que se encargan de podar para ellos mismos y para el resto de los habitantes del pueblo. En muchas localidades ya no hay gente que pode, pero en otras el conocimiento se ha transmitido de generación en generación. Cuando la posibilidad del monte ha superado la necesidad de los vecinos, los ayuntamientos han enajenado las cortas de leña, incluidas las leñas de poda.

\section{Objetivos}

Hemos pretendido documentar el conocimiento tradicional de la poda de roble en la comarca de Almarza, de manera sencilla. El objetivo es poder transmitir ese conocimiento a diversos colectivos:

- Vecinos que quieren podar ellos mismos sus "suertes".

- Adjudicatarios de aprovechamientos de poda.

- Agentes medioambientales.

- Técnicos forestales, tanto públicos como privados.

- Interesados en general. 
Para poder llegar a tan amplio espectro de personas, se necesita un documento muy fácil de interpretar. Por eso hemos redactado unas instrucciones sencillas y claras, acompañadas de dibujos explicativos.

\section{Resultados}

Este es un trabajo plenamente empírico. Pretendemos realizar ensayos para confirmar que este modo de ejecutar las podas es el más adecuado, pero antes de nada hemos considerado urgente documentar la experiencia de siglos, para que no se pierda este saber popular, al menos en nuestro pequeño ámbito geográfico.

En una breve introducción definimos trasmocho o árbol trasmocho como un árbol podado artificialmente al cual se le ha suprimido su guí, con la copa abierta en 3 a 5 ramas principales horizontales, de las que salen chupones que se cortan periódicamente para su aprovechamiento como leña.

Otras definiciones que se han incorporado al manual son las siguientes:

Un tirasavias es una rama horizontal o inclinada hacia abajo, que parte del brazo principal, y que se deja de cortar para ayudar al rebrote. Dejar suficientes tirasavias es fundamental para asegurar el brote posterior a la poda.

Las ramas de fuerza son ramas verticales que parten de un brazo o del mismo centro de la copa. Éstas absorben toda la fuerza de la brotación.

Se exponen las normas de poda para un caso general, de manera sencilla.

- La poda se ejecuta siempre a savia parada, hacia el final invierno, para favorecer la brotación posterior.

- El árbol a podar tiene que ser suficientemente vigoroso.

- Para provocar la reacción del árbol a la poda y generar una nueva copa de árbol trasmocho es necesario eliminar al menos el $80 \%$ de la superficie foliar.

- Se cortarán en primer lugar las ramas de fuerza, que son todos los brotes verticales. Entre los potenciales tirasavias que queden, se dejarán sin cortar algunos de los que no hayan sido dañados en el apeo anterior.

- En cada brazo hay que dejar al menos un tirasavias, preferentemente al final del mismo, para no comprometer la supervivencia del árbol.

- Los cortes deben hacerse en bisel para que no se acumule agua, a $5 \mathrm{~cm}$ de altura de la base de la rama en la parte más baja del bisel, y preservar todos los pequeños brotes y ramillas que hay en su base, porque funcionan como tirasavias que ayudan a la brotación.

- Siempre se tenderá a dejar más tirasavias en el lado de la umbría.

Estas normas están basadas en la experiencia acumulada por los vecinos, principalmente, y documentada a través de los agentes medioambientales. En otros lugares y dependiendo de las especies, se poda al final del verano, con fines de ramoneo. En nuestra comarca las podas no se autorizan antes del 1 de noviembre. De este modo la leña no está cargada de savia y tiene mejor calidad. 
No recomendamos las podas a vida o muerte, cuando los robles presentan poco vigor y no estamos seguros de su reacción positiva. Si el árbol no tiene reservas suficientes, el "esfuerzo" de una brotación de emergencia puede acabar secándolo.

Los árboles podados con excesiva precaución, en los que se elimina menos del $80 \%$ de la hoja, suelen tener una reacción pobre. El objetivo buscado es provocar brotes nuevos en las ramas principales del árbol, que generarán la leña en el futuro. Si no se poda con suficiente intensidad, la reacción del árbol será insuficiente.

Si se dejan ramas de fuerza, éstas absorberán toda la fuerza del brote, con lo que no se lograrán los objetivos. Por otra parte, los cortes de ramas gran diámetro no llegan nunca a cerrarse, pero si no se acumula el agua en ellos, se secan y no suponen inconveniente para el árbol. Incluso pueden llegar a sufrir pudriciones, pero el trasmocho es capaz de convivir con ellas, pues solo afectan a tejidos muertos.

El punto fuerte del manual son los dibujos explicativos de todos los conceptos expuestos. Sirva como ejemplo una muestra de ellos (Fig. 1, Fig. 2).

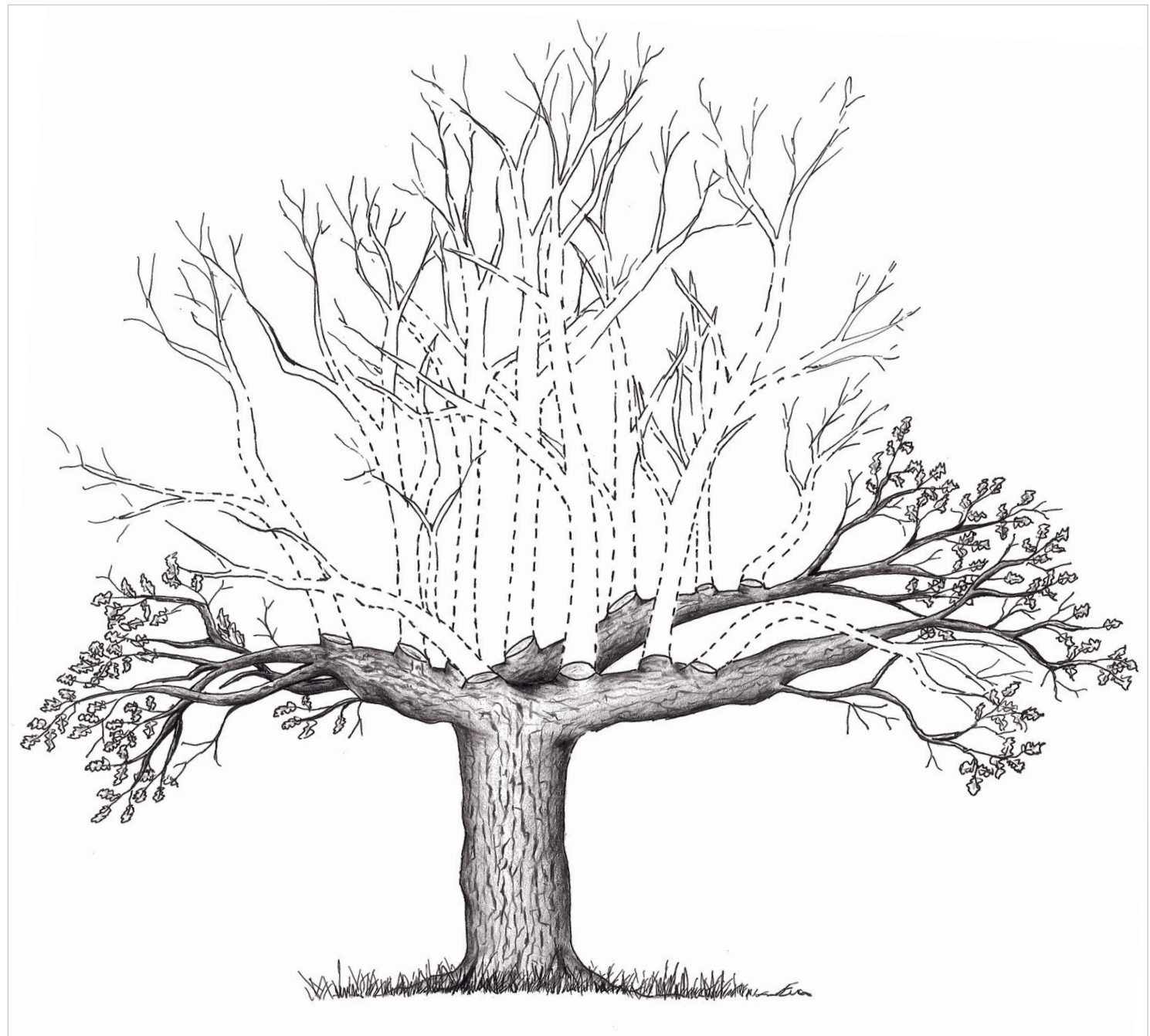

Figura 1. Forma típica de un roble (Quercus pyrenaica) podado "en turno". Se eliminan todas las ramas de fuerza y se deja suficiente número de tirasavias en cada brazo. 


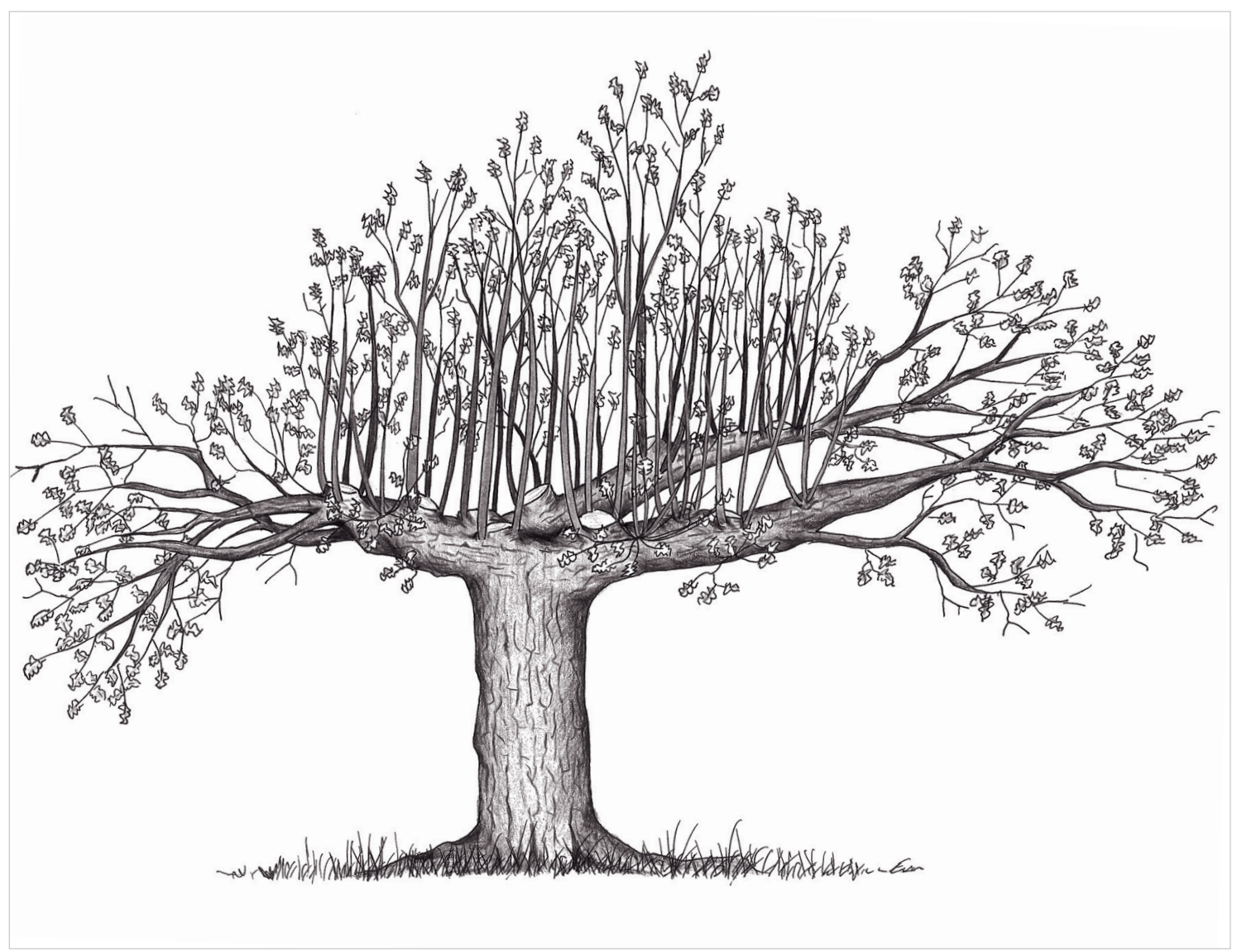

Figura 2. Objetivo perseguido con este tipo de podas: brotación abundante en el vaso que forman las ramas principales. Permite la obtención de leña posterior.

Además del caso general, hemos añadido tres casos de poda de árboles muy frecuentes en nuestras dehesas:

- Árboles que debían haber sido podados hace años (pasados de turno). Hay grandes diferencias entre los brotes verticales, porque unos se han apoderado de otros. Los diámetros en base de los brotes dominantes son considerablemente mayores.

No importa cortar los brotes dominantes, a no ser que superen los $50 \mathrm{~cm}$ en la base, en cuyo caso se debe elevar la hasta donde la rama alcance un diámetro de $40 \mathrm{~cm}$. No subir más de $1 \mathrm{~m}$, porque a mayor altura ya no es posible la ejecución de la poda posterior.

- Árboles que no han sido correctamente podados previamente.

En las podas anteriores se dejaron ramas de fuerza sin podar (mal seleccionadas) que han alcanzado grandes dimensiones y que perjudicaron el brote posterior. Apenas hay otras ramas aprovechables.

En ese caso se deben cortar estas ramas principales a cierta altura (todas a 
la misma altura más o menos) dejando como tirasavias algún ramillo, aunque tenga poca entidad.

- Árboles con riesgo de desgarro.

Si hay ramas que por su excesivo peso pueden provocar la rotura del árbol, es necesario reducir la longitud de los brazos. El desgarro es el mal a evitar. En ese caso, podemos cortar diámetros de hasta $45 \mathrm{~cm}$, pero siempre que quede un tirasavias adecuado en el extremo del brazo.

Por último, el manual termina con unas indicaciones para la "metida en turno" de nuevos trasmochos; es decir, la forma de hacer el corte a la guía principal del árbol para permitir su conversión en trasmocho y destinarlo a la producción de leña.

\section{Discusión y conclusiones}

Las formas de masa culturales, como las dehesas de roble, son un legado histórico cuyo valor es incontestable. Además de su inestimable valor desde el punto de vista paisajístico, destacan como formaciones biodiversas, por la cantidad de nichos ecológicos diferentes que proporcionan. En algunas zonas de la provincia de Soria se ha seguido transmitiendo el conocimiento de la forma tradicional de podar, de padres a hijos, y hemos considerado muy interesante documentarlo.

Si hay personas, tanto vecinos como adjudicatarios de aprovechamientos forestales, que quieran podar los robles y no cuenten con la suficiente experiencia, aquí tienen una herramienta que les puede ser de utilidad.

No hemos difundido suficientemente el manual, por lo que aún no podemos valorar su acogida. No obstante, creemos que puede ser una herramienta útil para los colectivos implicados.

Nuestro propósito es que este documento vaya experimentando revisiones que lo vayan mejorando, y adecuando a los fines propuestos.

A partir de ahora, pretendemos ir sistematizando las investigaciones con diferentes ensayos de intensidad de poda, frecuencia, diámetros máximos de corte, etc. Revierte gran interés el análisis de la influencia de las ramas de fuerza sobre la reacción del árbol a la poda. Se están ensayando las podas en dos tiempos: en árboles en los que se dejaron muchas ramas de fuerza y el brote no fue suficientemente abundante, se apean posteriormente todas esas ramas y se analiza la respuesta del árbol.

Es probable que dentro de algunas décadas ya nadie corte leña para consumo doméstico. Quizás entonces se realice el trasmocho como forma de conservación de los árboles, por evitar la pérdida de los paisajes y de los ecosistemas asociados. Quizás, por el contrario, la sociedad no perciba entonces estos como un valor, y se deje morir a los viejos trasmochos una vez cumplida su misión productiva.

Si continúa habiendo interés por parte de algunas personas de podar robles, y en especial, por parte de los pocos habitantes que quedan en nuestros pueblos, les animaremos a ello. 


\section{Agradecimientos}

A Cándido Andrés por promover la redacción del manual y a Eva González por la realización de los dibujos. A todos los agentes medioambientales de la Sección Territorial Urbión - Cebollera de Soria, por sus aportaciones y su inquietud.

\section{Bibliografía}

Allende, F., Gómez Mediavilla, G., López Estébanez, N., Sobrino García, J., 2013. Podas y trasmochos en las ordenanzas forestales del Sistema Central español y su impronta en el paisaje forestal actual. Cuad. Soc. Esp. Cienc. For. 38, 43-49.

Aragón Ruano, A., 2009. Los robles trasmochos guiados o ipinabarros: una apuesta sostenible de futuro para una técnica forestal olvidada. Cuad. Soc. Esp. Cienc. For. 30, 137142.

Cantero, A., Passola, G., 2013. ¿Qué son los trasmochos? In: Apuntes sobre trasmochos. Guía de buenas prácticas para el trasmocheo. Diputación Foral de Guipúzcoa, pp. 1128.

Francisco, M.D., 2013. Los trasmochos en el siglo XXI. In: Apuntes sobre trasmochos. Guía de buenas prácticas para el trasmocheo. Diputación Foral de Guipúzcoa, pp. 49-60.

Martínez Rodríguez, L., 2014. La gestión de los recursos comunales y la igualdad en la comunidad de San Andrés de Soria. Tesis doctoral. Universidad de Barcelona. 